\title{
13 Self-injury response and intervention policy
}

\author{
Emily Berger and Janis Whitlock
}

\section{How to use this policy}

The purpose of this chapter is to provide an outline of a policy addressing productive responses in schools to students who have self-injured. This policy example is intentionally broad and school policy creators are encouraged to delete and build upon suggestions below to create a policy that best represents their school.

[To adapt and use this policy, delete, or modify the text as indicated]

\section{[INSERT name of school] self-injury response and intervention policy}

\section{Rationale}

Non-suicidal self-injury (otherwise referred to as self-injury and deliberate self-harm [DSH]) refers to deliberate actions to harm one's own body (Nock \& Favazza, 2009). Behaviours include self-inflicted cutting, burning, hitting, scratching, and any other behaviours that cause harm to a person's body (Martin et al., 2010). Educators may witness and be the first responder to a student who has self-injured, may have a student disclose acts of self-injury to them, or may need to monitor a student in their class who has a history of the behaviour (Berger et al., 2014a; 2014b).

An estimated 7.5 to $46.5 \%$ of adolescents have engaged in self-injury at least once (Cipriano et al., 2017; Martin et al., 2010). Self-injury is prevalent among males and females (Jacobson \& Gould, 2007). People with a history of mental illness, with prior engagement in self-injury, who have family members or friends who have self-injured, who abuse drugs or alcohol, or who have a history of trauma or physical or sexual abuse are at greatest risk of engaging in self-injury (Toste \& Health, 2010).

There are a number of reasons that people may self-injure; regulating unwanted thoughts and emotions is understood as a primary underlying goal (Nock \& Favazza, 2009). A growing body of research suggests that the physical pain and relief that follows self-injury essentially tricks the 


\section{Berger \& Whitlock}

brain into perceiving relief from emotional pain (Franklin, 2014). Selfinjury can also be contagious, meaning that youth adopt the practice after observing it in other people or through media (Toste \& Health, 2010).

\section{Purpose}

This school policy aims to assist educators in identifying and sensitively responding to self-injury in school settings. Despite the fact that self-injury and suicide thoughts and behaviours (STB) are distinct phenomena - one is undertaken to feel better (self-injury) and the other undertaken to end the capacity to feel at all (STB) - they can co-occur (Owens et al., 2002). Indeed, self-injury is a risk factor for STB and so should trigger a suicide risk assessment. That said, since non-suicidal self-injury should not automatically trigger the same emergency protocols that STB often triggers, self-injury and STB school policies should be distinct.

\section{Scope}

This policy applies to all school staff team members, including teachers, school leaders, and the school mental health worker(s) for [INSERT name of school], and parents/carers and students of [INSERT name of school] when responding to students who have self-injured.

[INSERT specific roles and responsibilities of individuals or teams]

\section{Policy statement}

This school policy aims to address the issue of non-suicidal self-injury and is based on extant templates in the areas of non-suicidal self-injury and deliberate self-harm (DSH). The following policy components are focused specifically on self-injury but also address the increased risk of STBs for self-injuring adolescents.

\section{Roles and responsibilities of teachers and other school staff}

The following table delineates the roles and responsibilities of all school staff at [INSERT name of school]. Identification of a school crisis team or point people is appropriate for schools who do not have full-time access to a school nurse or school mental health staff member (Bubrick et al., 2010; Walsh, 2012). These point people or members of the crisis team will be educators and school staff with specialist and ongoing training in responding to and managing instances of student self-injury and mental illness. 


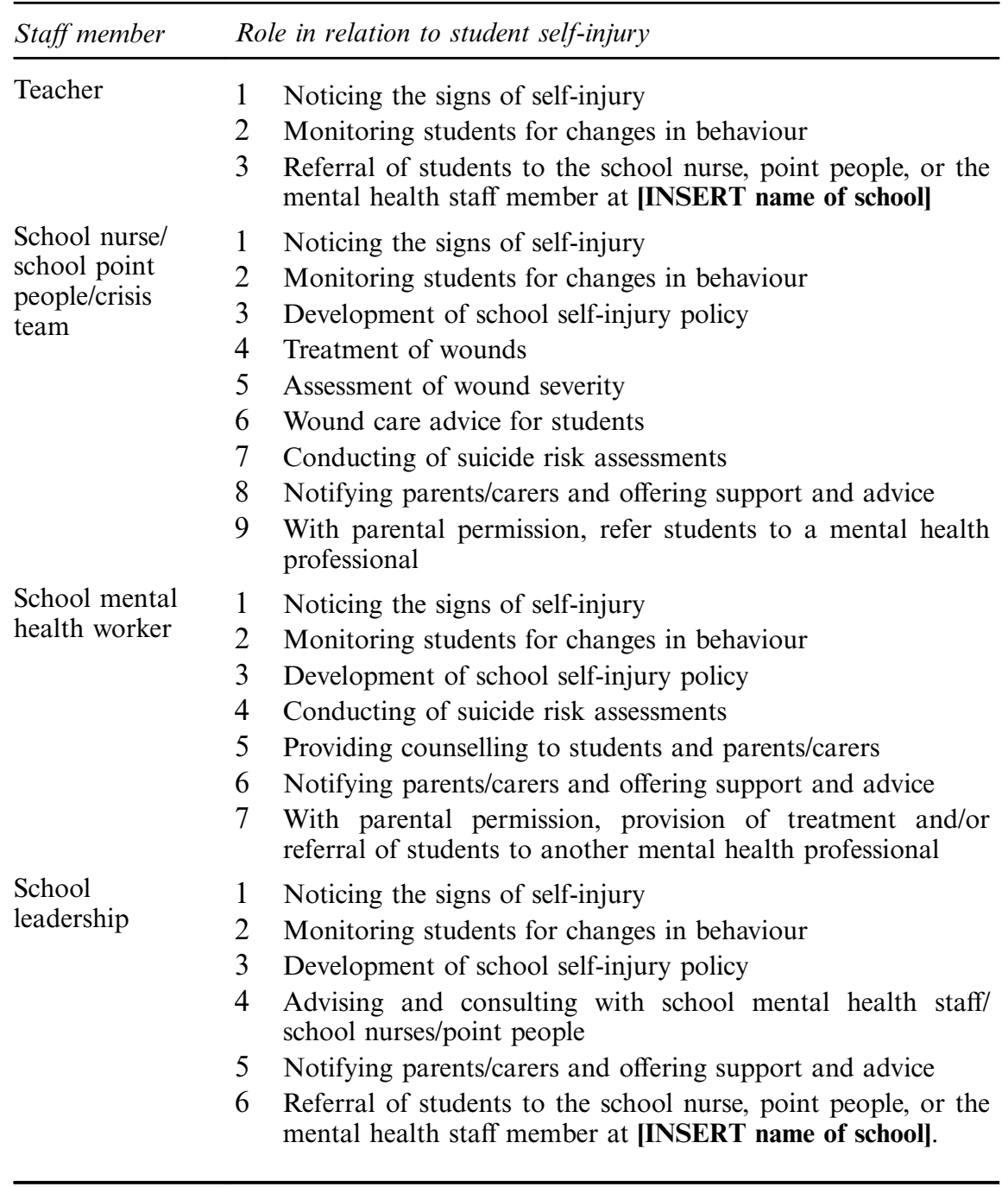

\section{Professional development for school staff}

Teachers and school leaders require training to be able to respond to and refer students if they witness self-injury or if a student discloses self-injury. School nurses, mental health staff, and school crisis teams/point people should receive additional training to conduct a suicide risk assessment, assess the psychological and/or physical health of the self-injuring student, contact the student's parents or carers, and refer the student to a mental health service or accompany the student to the hospital emergency department (Berger et al., 2014b; Walsh, 2012). 


\section{Berger \& Whitlock}

\section{Witnessing a student self-injuring}

School staff at [INSERT name of school] who witness a student self-injuring should respond using a calm, compassionate, and non-judgemental approach. School staff should avoid showing anger, horror, panic, frustration, or disgust (Toste \& Health, 2010). Adopting a low key, dispassionate demeanour and asking questions based on respectful curiosity is the central pillar of student-staff interactions related to self-injury (Walsh, 2012). School staff should not move directly into attempting to fix or otherwise solve student problems and should not deny student feelings (Berger et al., 2015). Self-injury implements (e.g., blade or lighter) should be removed using a calm and compassionate manner, and the staff member should stay with the student until another member of school staff (preferably a school nurse or mental health worker/point person) can attend to the student (Berger et al., 2015). If there are open wounds that need attention, the first contact staff member may escort the student to the school nurse/point person/crisis team at [INSERT name of school] for treatment of the wounds (see section on wound care and harm minimisation) (Bubrick et al., 2010; Onacki, 2005).

\section{Noticing signs that a student has self-injured}

The following are signs that teachers or other school staff might notice if a student engages in self-injury. Because of feelings of shame or guilt, fear of negative reactions, and/or because self-injury is used for coping, students may avoid seeking help (Berger et al., 2013). Signs that a student has selfinjured include:

a Unexplained scars and wounds which may include cuts, burns, scratches, or bruises

b Students wearing clothing with long sleeves and pant legs. This behaviour is particularly concerning when the weather is warmer and students do not want to participate in sporting and swimming activities

c Students engaging in secretive behaviour and withdrawing from other people

d Students searching online or writing and drawing about self-injury in school essays, journals, and artwork

e A student showing disregard for their own physical safety and health, their hygiene, or their personal appearance (Berger et al., 2015; Bubrick et al., 2010; Toste \& Health, 2010; Walsh, 2012).

\section{Monitoring the student following self-injury}

Students who have a history of self-injury should be monitored for changes in their behaviour. The previously presented warning signs may be used to monitor these students (see section on noticing signs that a student 
has self-injured). The other purpose of monitoring students who have a history of self-injury is due to the likelihood of self-injury reoccurring and the emotional, mental, and physical consequences of self-injury (e.g., infection, scarring, anxiety, shame, or depression) (Bubrick et al., 2010; Toste \& Health, 2010).

\section{Wound care and harm minimisation}

School nurses, mental health personnel, and point people/crisis team members should discuss with students who self-injure how they can care for their wounds, how students identify when a wound is not healing, and when students may be experiencing physical complications from their injuries. Harm minimisation also involves conversations with students about how sterile the implements are that they use to self-injure. How to approach this conversation with students is detailed here:

- Use neutral, non-judgemental language

- Use pragmatic and matter-of-fact questioning

- Use the student's language for their wounds, scars, and self-injury

- Allow the student space and time to discuss their self-injury, wounds, and scars

- Listen to the students without an agenda or attempting to solve their problems

- Address any questions from the students about self-injury

- Refer to what you know about self-injury more generally and enquire if this is the same or not for the student.

\section{Self-injury contagion in schools}

Contagion of self-injury in schools can be addressed in a number of ways:

a Deliberately showing wounds and scars should not be permitted by students at school

b Students should be educated about the risks of contagion of self-injury and the importance of not showing their scars and wounds to prevent peers from adopting the behaviour

c Discussing self-injury as one of many maladaptive coping strategies and discussing adaptive methods of coping with students

d Telling students that all maladaptive behaviours (including self-injury) require help from an adult and identifying which adult they can speak to if they suspect or know of a peer that has self-injured or if they themselves have self-injured

e School staff should receive training regarding how to compassionately speak to students about displaying wounds, while also not increasing the student's sense of shame or excluding students from school or 
school activities (e.g., sporting events) (Toste \& Health, 2010; Walsh, 2012).

\section{Engaging with parents and carers}

Offering support and advice to parents/carers about the risks associated with self-injury, the facts about self-injury, and how parents can respond to their self-injuring child is the role of the school nurse, school mental health staff member, school crisis team or point people, and members of the school leadership team at [INSERT name of school]. Advising parents and caregivers on how and when to engage with mental health services and specialists external to [INSERT name of school] is also the role of these members of staff. The decision to inform parents/carers about the self-injury is based on the student's level of risk for further self-injury and STBs, the severity of the injuries caused by the student when self-injuring, the potential risk posed to students by notifying the parents/carers, and the need for a referral to a mental health service external to [INSERT name of school] (Bubrick et al., 2010; Toste \& Heath, 2010; Walsh, 2012). A notification to child protection services is required if parents/carers repeatedly and without explanation fail to enact the advice of health professionals (Walsh, 2012).

\section{Conducting a suicide risk assessment}

A suicide risk assessment should be conducted at by [INSERT name of school] school nurse, mental health professional(s), or point people/crisis team with students who have been identified as engaging in self-injury (refer to [INSERT name of school] policy on suicide). An assessment of the student's risk for further self-injury and their mental health should also be conducted and treatment should be provided to the student based on the outcome of this assessment (Toste \& Health, 2010). Suicide and self-injury risk assessments should continue to be conducted with students who have a history of either of these behaviours or with students who continue to express thoughts related to suicide and/or self-injury.

\section{Feedback loop and self-care}

Support and feedback should be provided to the referring teacher or school staff team member, as long as the confidentiality of the student is maintained (Berger et al., 2015; Walsh, 2012). To maintain the confidentiality of the student, this feedback may take the form of informing the referring teacher or staff member that they responded correctly to the student and/or by providing strategies for staff regarding how they might respond to students who selfinjure in the future. 


\section{Rating of evidence base}

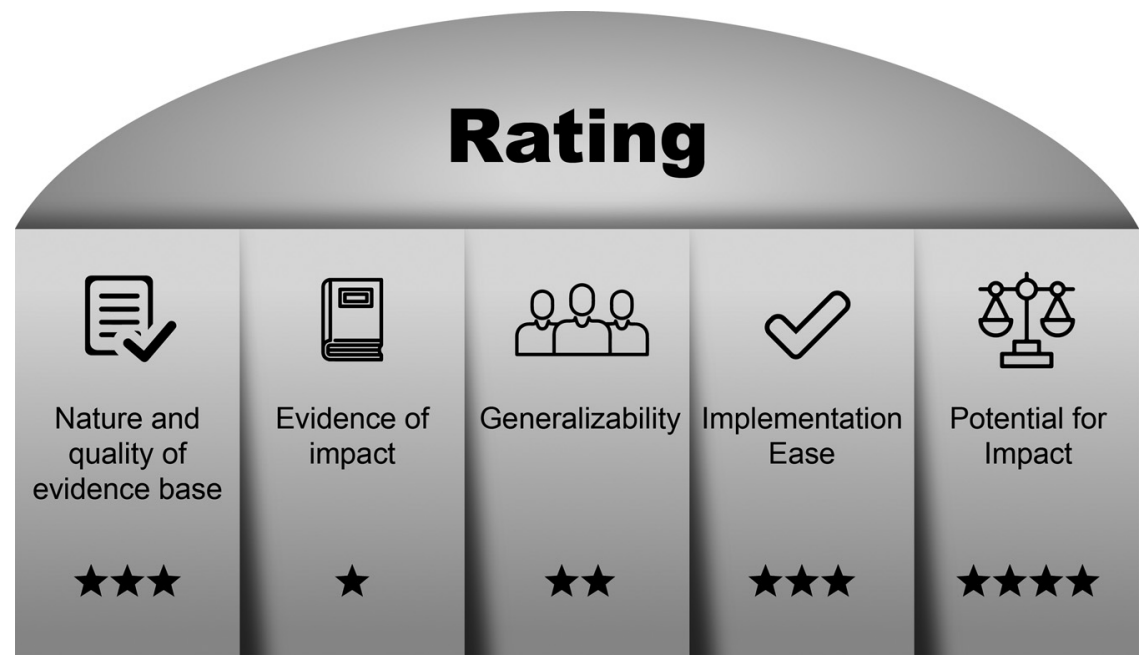

Figure 13.1. Self-injury Response and Intervention Rating of Evidence.

Author Note. There is a good evidence-base for the recommendation included in this policy. The results of this evidence-base are consistent and with a low risk of bias. The research evidence used in this policy demonstrates levels of impact that are not known. The research used in this policy is generalizable and representative of the broader population of young people who self-injure and across schooling contexts. Self-injury school policy requires a mental health professional, school nurse, point person/crisis team to be available and for all school staff to have training and awareness of student self-injury and how to respond. A school self-injury policy is able to minimise or prevent further student self-injury and to equip school staff to manage this behaviour in schools. Appropriate management of self-injury in schools will help to facilitate student help-seeking for peers or themselves for self-injury.

\section{Authorship}

Dr Emily Berger, Monash University

Dr Janis Whitlock, Cornell University

[INSERT RELEVANT STAFF MEMBERS]

\section{Related policy and documents}

\section{[INSERT RELEVANT POLICY AND DOCUMENTS]}

Teacher wellbeing

Mental health promotion

Suicide

Risk assessment

Mandatory reporting 


\section{Date of ratification}

This policy was ratified on the [INSERT DATE].

\section{Date of Review}

This policy will be reviewed by [INSERT DATE].

\section{Further reading}

Nock, M. K. (Ed.). (2009). Understanding non-suicidal self-injury: Origins, assessment, and treatment. American Psychological Association.

Walsh, B. W. (2012). Treating self-injury: A practical guide (2nd ed.). The Guilford Press.

\section{Expert consultation}

Consultation with mental health providers and services is recommended to enact this policy. A crisis team/point people and expert mental health personnel are required to assess, manage, refer, and/or provide treatment to students who have self-injured or who are reported or suspected of self-injury.

\section{References}

Berger, E., Hasking, P., \& Reupert, A. (2013). "Listen to them": Adolescents' views on helping young people who self-injure. Journal of Adolescence, 36(5), 935-945.

Berger, E., Hasking, P., \& Reupert, A. (2014a). "We're working in the dark here": Education needs of teachers and school staff regarding student self-injury. School Mental Health, 6(3), 201-212.

Berger, E., Hasking, P., \& Reupert, A. (2014b). Response and training needs of school staff towards student self-injury. Teaching and Teacher Education, 44, 25-34.

Berger, E., Hasking, P., \& Reupert, A. (2015). Developing a policy to address nonsuicidal self-injury. Journal of School Health, 85(9), 629-647.

Bubrick, K., Goodman, J., \& Whitlock, J. (2010). Non-suicidal self-injury in schools: Developing and implementing school protocol. The fact sheet series. Cornell Research Program on Self-Injury and Recovery. http://www.selfinjury.bctr.cornell. edu/documents/schools.pdf

Cipriano, A., Cella, S., \& Cotrufo, P. (2017). Non-suicidal self-injury: A systematic review. Frontiers in Psychology, 8, 1946.

Franklin, J. (2014). How does self-injury change feelings? The fact sheet series. Cornell Research Program on Self-Injury and Recovery. http://www.selfinjury.bctr.cornell. edu/perch/resources/how-does-self-injury-change-feelings-5.pdf

Jacobson, C. M., \& Gould, M. (2007). The epidemiology and phenomenology of nonsuicidal self-injurious behavior among adolescents: A critical review of the literature. Archives of Suicide Research, 11(2), 129-147. 
Martin, G., Swannell, S., Harrison, J., Hazell, P., \& Taylor, A. (2010). The Australian national epidemiological study of self-injury (ANESSI). https://www.familyconcern publishing.com.au/wp-content/uploads/2016/06/ANESSI.pdf

Nock, M. K., \& Favazza, A. R. (2009). Non-suicidal self-injury: Definition and classification. In M. K. Nock (Ed.), Understanding non-suicidal self-injury: Origins, assessment, and treatment (pp. 9-18). American Psychological Association.

Onacki, M. (2005). Kids who cut: A protocol for public schools. Journal of School Health, 75(10), 400-401.

Owens, D., Horrocks, J., \& House, A. (2002). Fatal and non-fatal repetition of self-harm. British Journal of Psychiatry, 181, 193-199.

Toste, J. R., \& Heath, N. L. (2010). School response to non-suicidal self-injury. The Prevention Researcher, 17(1), 14-17.

Walsh, B. W. (2012). Treating self-injury: A practical guide (2nd ed.). The Guilford Press. 